\title{
PROCESO DE ENFERMERÍA PARA GRUPOS DE AYUDA MUTUA: UNA PROPUESTA DESDE EL MODELO DE PROMOCIÓN
}

\author{
NURSING PROCESS FOR MUTUAL AID GROUPS: A PROPOSAL FROM THE PROMOTION MODEL
}

OLGA BARRAGÁN HERNÁNDEZ* EVA ANGELINA HERNÁNDEZ VILLA** ROCIO ALEJANDRA FLORES LEÓN** MARÍA RUBI VARGAS***

\section{ABSTRACT}

This article discusses the ways of evaluation by the teaching staff in the field of clinical practice in nursing, and the teacher is the one who develops strategies of teaching - as needed basis, to guide students to make their own learning for development of a professional future. It aims to see in a general way the process of learning assessment in clinical practice that made teachers summarized in three categories: academic performance of students, evaluation of products (results of teaching), and search standardizable attributes or traits, these methods are adapted according to the clinical area where the student develops.

It can be an excellent professional without being therefore an excellent teacher and therefore no longer be an excellent evaluator that considers three main causes of errors in the evaluation process: Insufficient information halo effect, or tendency to establish categories and stereotypes error generosity, giving them a preamble to a hazard assessment invalid and unreliable bringing consequences of psychological, physical and social environment for nursing students.

Keywords: evaluation, clinical practice, teaching - student nurse, nursing teacher.

\section{RESUMEN:}

I ntroducción: La presencia de enfermedades crónicas no transmisibles representa en la actualidad problemas emergentes de salud pública, que aparecen como resultado de los cambios sociales y económicos que modificaron el estilo de vida de una gran parte de la población. Situación que requiere de la participación de un equipo multidisciplinario adiestrado, que proporcione las herramientas de autocuidado que los integrantes de los grupos de ayuda muta (GAM) requieren.

Desarrollo: Es necesaria una educación participativa que considere al enfermo crónico generador de su propio aprendizaje a través de los GAM, que nacen como una estrategia de autocuidado, con la ayuda del equipo de salud y las experiencias de sus compañeros en relativa igualdad de condiciones. Se plantea el Modelo de promoción de la salud de Nola Pender como marco para desarrollar un proceso de enfermería que integre los diagnósticos de enfermería que sirvan de base para planificar las estrategias de promoción a la salud con el propósito de ayudar a los integrantes de los GAM a modificar su estilo de vida.

Conclusiones: La propuesta desde la perspectiva del MPS e implementada a través del proceso de enfermería, ofrece la oportunidad de contribuir a la modificación de estilos de vida y por consiguiente a la mejora en la calidad de vida de los integrantes de los GAM.

Palabras clave: Grupos de ayuda mutua, Proceso de enfermería, Modelo de promoción de la salud (DeCS, BIREME).

\footnotetext{
* Maestra en Ciencias. Docente de Tiempo Completo. Departamento de Enfermería. Universidad de Sonora. Correo electrónico: barragan@enfermeria.uson.mx

** Licenciada en Enfermería. Egresada del Departamento de Enfermería de la Universidad de Sonora.

*** Doctora en Ciencias de enfermería. Docente del Departamento de enfermería de la universidad de Sonora.
} 


\section{INTRODUCCIÓN}

El perfil epidemiológico de México está caracterizado por la presencia de enfermedades crónicas no transmisibles (ECNT) como la diabetes, hipertensión arterial, entre otras, donde el sobrepeso y la obesidad representan un factor de riesgo importante para la adquisición de estas enfermedades de la etapa postransicional. Además, la existencia de un rezago epidemiológico con enfermedades infecciosas que hace aún más compleja la atención a la salud de la población (1).

Esta situación representa un desafío para los prestadores de servicios de salud como los profesionales de enfermería que consideran la educación a la salud como una excelente opción de intervención, pues la enfermería es una profesión con características eminentemente sociales, vinculada directamente a la población y comprometida con la atención de sus necesidades (2).

En este contexto, la promoción de la salud surge como herramienta en la declaración de Atención Primaria para la Salud, en Alma Ata Rusia en 1978, donde se valora la educación como metodología válida para la formación de las personas en estilos de vida saludables, con el fin de crear hábitos y costumbres sanas, cuya práctica cotidiana fortalezca las condiciones para mejorar la calidad de vida necesaria para un desarrollo humano adecuado, en lo individual y colectivo (3).

En el marco del desarrollo de modelos y teorías en Enfermería, Nola J. Pender desarrolló el Modelo de Promoción a la Salud (MPS) ${ }^{(4)}$ con el que propone comprender los comportamientos humanos relacionados con la salud, y a su vez, orienta hacia la generación de conductas saludables. Establece que la conducta está motivada por el deseo de alcanzar el bienestar y el potencial humano, por lo que crea un modelo de enfermería que diera respuestas a la forma cómo las personas adoptan decisiones acerca del cuidado de su propia salud ${ }^{(5)}$. Además, se puede vincular con herramientas generadas en el extranjero que se han adaptado al contexto de la enfermería en México tales como la Clasificación de Diagnósticos Enfermeros (NANDA) ${ }^{(6)}$, Clasificación de Resultados Esperados (NOC) ${ }^{(7)}$ y Clasificación de Intervenciones de Enfermería (NIC) ${ }^{(8)}$.

En este contexto, la enfermería como disciplina profesional mantiene como propósito utilizar todas las capacidades y potencialidades del individuo para la mejora en su calidad de vida, para lo cual aplica el PE, que se define como una herramienta metodológica que promueve la utilización del pensamiento crítico, es proactivo, dinámi- co y centrado en las respuestas humanas, en el manejo de los riesgos y la promoción de la salud, haciendo frente a los problemas de salud reales y potenciales. ${ }^{(9)}(10)$, a través de la vinculación de las taxonomías NANDA, NOC, NIC favoreciendo la homologación del lenguaje dentro de la práctica profesional, generando en la enfermera (o) capacidades para el razonamiento clínico y la toma de decisiones y por ende la autonomía en el ejercicio de la profesión. Con la aplicación del PE los profesionales de enfermería brindan el cuidado de manera racional, lógica y sistemática. Los planes de cuidados se han convertido en el instrumento clínico que sirve para registrar el proceso de cuidado individualizado utilizando como base modelos estandarizados o no ${ }^{(11)}$.

Por otro lado, es la promoción de la salud la estrategia adecuada para la capacitación de los integrantes de los grupos de ayuda muta (GAM) para el control de las ECNT ya que proporciona las herramientas de autocuidado a la salud a través de la prevención, tratamiento y control de dichos padecimientos ${ }^{(12)}$

En estos espacios, el profesional de enfermería puede intervenir de manera independiente en la educación de los integrantes, utilizando como herramienta de trabajo la metodología del PE.

Dado lo anterior el presente trabajo tuvo como objetivo elaborar una propuesta para la aplicación del PE para los GAM, desde la perspectiva del modelo de promoción de la salud de Nola J. Pender.

\section{DESARROLLO}

\section{El Modelo de Promoción de la Salud}

El MPS posee dos sustentos teóricos: la teoría de aprendizaje social de Albert Bandura y el modelo de valoración de expectativas de la motivación humana de Feather ${ }^{(5)}$, con el MPS de Nola J. Pender, el PE se orienta y aplica hacia el desarrollo de conductas saludables o conductas que favorecen la salud, al tiempo que motiva el estilo de vida saludable en la gente joven y adulta ${ }^{(12)}$.

El MPS es un esquema que integra constructos para detectar la estructura multidimensional de los rasgos individuales que, en interacción con su ambiente, mejoran los resultados asociados a salud. La conducta promotora de salud (resultado conductual) está influenciado por diferentes conceptos, como las características y experiencias individuales previas, por cogniciones y afectos relativos a comportamientos específicos ${ }^{(13)}$. 
El primer concepto se refiere a los antecedentes o experiencias que pudieran tener efectos directos e indirectos en el compromiso del usuario con las conductas promotoras de la salud y a los factores personales que se clasifican como biológicos, psicológicos y socioculturales, que de acuerdo al enfoque del MPS son predictivos de cierta conducta y están condicionados por la naturaleza de la meta deseada ${ }^{(5)}$.

El segundo concepto incluye los principales componentes del modelo, que abarca desde los conocimientos, sentimientos y emociones hasta las creencias específicas que posee la persona sobre la conducta promotora deseada (5). "El modelo revisado, que se está demostrando de forma empírica actualmente, identifica los conceptos importantes para las conductas de promoción de la salud y facilita la generación de las hipótesis demostrables" (14) (Figura 1).

Figura 1. Modelo de Promoción de la Salud de Nola J. Pender modificado.

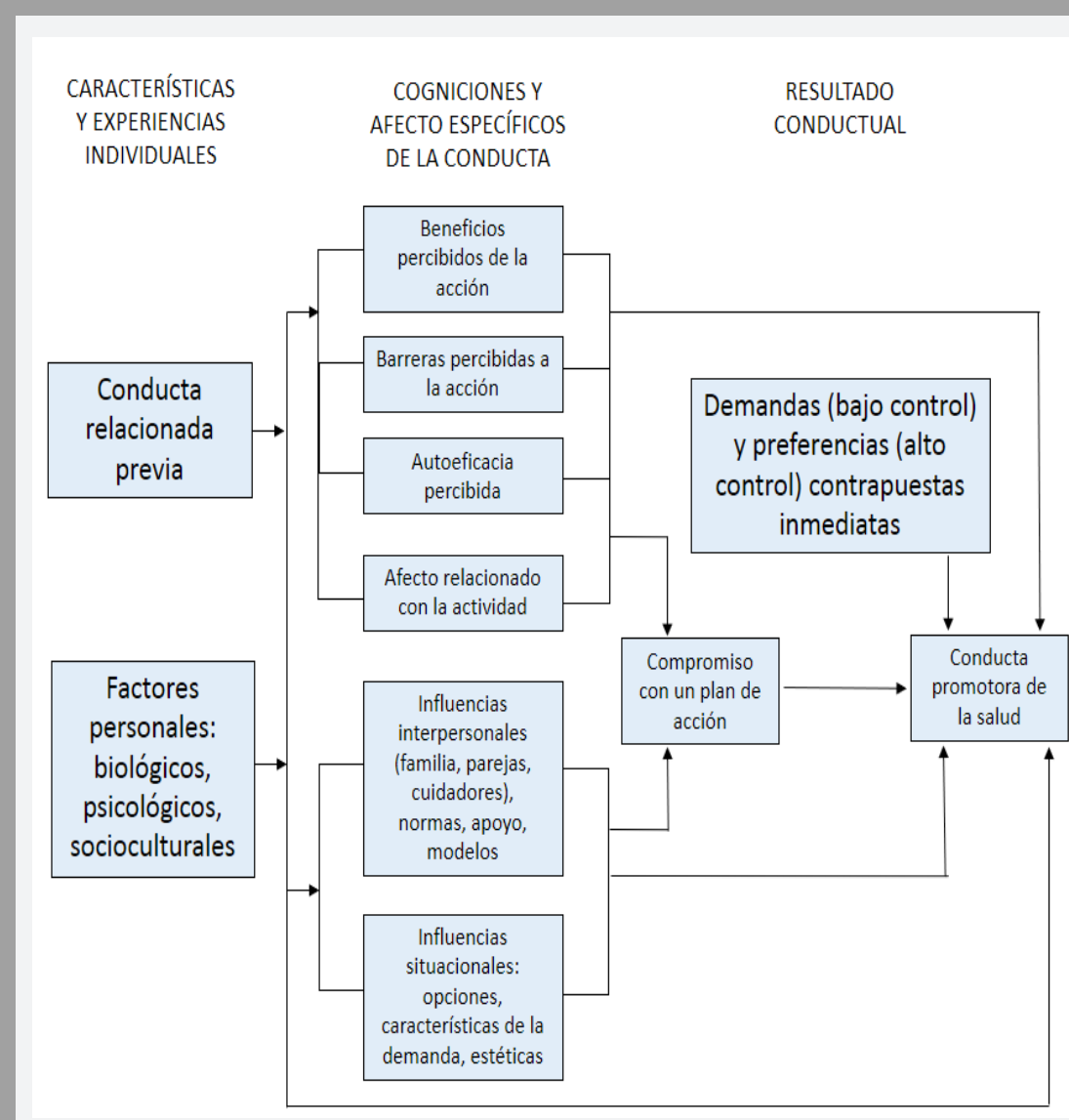

Fuente: Alligod, M. (2015). Modelos y teorías en enfermería (8a ed.). Barcelona, España : Elsevier.
Propuesta para la aplicación del proceso de enfermería desde el MPS.

Los GAM se constituyen en escenarios para la capacitación voluntaria y periódica de los enfermos crónicos con énfasis en el manejo no farmacológico y el autocuidado de la salud, lo que contribuye a hacer más efectiva la intervención de enfermería con programas de educación para la salud, reforzando la adhesión al tratamiento y el cumplimiento de las prescripciones del equipo de salud, permitiendo además el intercambio de experiencias entre los enfermos, facilitando la adopción de los cambios conductuales requeridos para el control de las enfermedades.

La participación de enfermería en los GAM consiste en la aplicación del PE, donde se pretende que el profesional concentre lo esencial de sus conocimientos para ayudar a resolver los problemas de salud de los enfermos que integran estos grupos y promover así su calidad de vida. Como todo método sus etapas son sucesivas y se relacionan entre sí, haciendo de la ejecución una práctica humanizada e integral, respetando ante todo al enfermo y a su familia. Es a partir de la aplicación del PE que se identifican los problemas reales y potenciales (diagnósticos de enfermería), estableciendo planes de cuidados individualizados para resolverlos ya sea previniendo o controlando las ECNT, como diabetes e hipertensión arterial entre otras.

Desde la dinámica de los GAM se abordan los conceptos del MPS. En primer lugar, las características y experiencias previas de los usuarios se incluyen desde los datos sociodemográficos del usuario, antecedentes patológicos y no patológicos, estado de salud actual (ECNT que padece actualmente), conductas que ha tenido antes de integrarse al GAM, tanto positivas como negativas, en relación a su enfermedad, es decir, el cumplimiento del régimen terapéutico, la búsqueda fallida de cambios en su 
estilo de vida hasta ese momento e inclusive la integración previa a un grupo de este tipo.

Por otro lado, las cogniciones y afectos específicos de la conducta que deberán valorar en el participante incluyen las barreras que percibe para el logro de una conducta promotora de la salud, los beneficios que este cree que tendrá dicha conducta en su salud, la capacidad que considera que tiene para lograr un cambio en su estilo de vida y las posibles influencias que podrían tener efecto sobre dicha conducta, es decir, si cuenta con el apoyo de su familia o sus círculos sociales más cercanos, e incluso si la situación actual en la que vive es favorable para el logro de las metas que éste usuario se proponga en relación a la mejora de su salud durante su participación en el GAM

Desde esta perspectiva, la valoración de enfermería, es una etapa clave porque recopila los datos sobre el estado de salud de las personas (que conforman los GAM) a través de diversas fuentes (primaria y secundarias) utilizando los siguientes métodos: entrevista, observación y examen físico; donde el primero tiene una importancia significativa porque recoge información específica y necesaria para los diagnósticos de enfermería y la planificación de los cuidados, los cuales incluyen datos como: síntomas, conductas, sentimientos, manifestaciones verbales o no verbales, entre otros, como consecuencia de su estado de salud, que se confrontan con los datos observables y los obtenidos a través del examen físico, buscando su validación y organizándolos

En el caso particular de los grupos de ayuda mutua, la conducta promotora esperada de los participantes será su asistencia constante al grupo, el cumplimiento de las indicaciones médicas y de enfermería, el control a través de los exámenes de laboratorio, la modificación de prácticas alimenticias inadecuadas para la salud, la disposición para un cambio de estilo de vida que influya de manera directa en su salud, es decir, que el usuario se vuelva gestor de su propia salud.

El profesional de enfermería después de construir una base de datos (sobre el estado de salud de la persona) validada y organizada, emitirá un diagnóstico de enfermería gracias al cual será posible elaborar un plan de cuidados donde los diagnósticos de enfermería reales, de riesgo y de promoción de la salud sirven de base para el diseño de estrategias de educación para la salud que ayuden a cada integrante de los GAM a lograr el resultado conductual esperado, donde se tomen en cuenta, en primer lugar el compromiso de los usuarios para participar de manera activa en el GAM, que logren identificar cuáles son las necesidades que tienen actualmente (demandas) y sus preferencias en cuanto a las metas que se plantee, con el fin de lograr una conducta promotora de la salud benéfica para cada usuario de manera individual y para todo el grupo.
Figura 2. Esquema aplicado de MPS a los GAM.

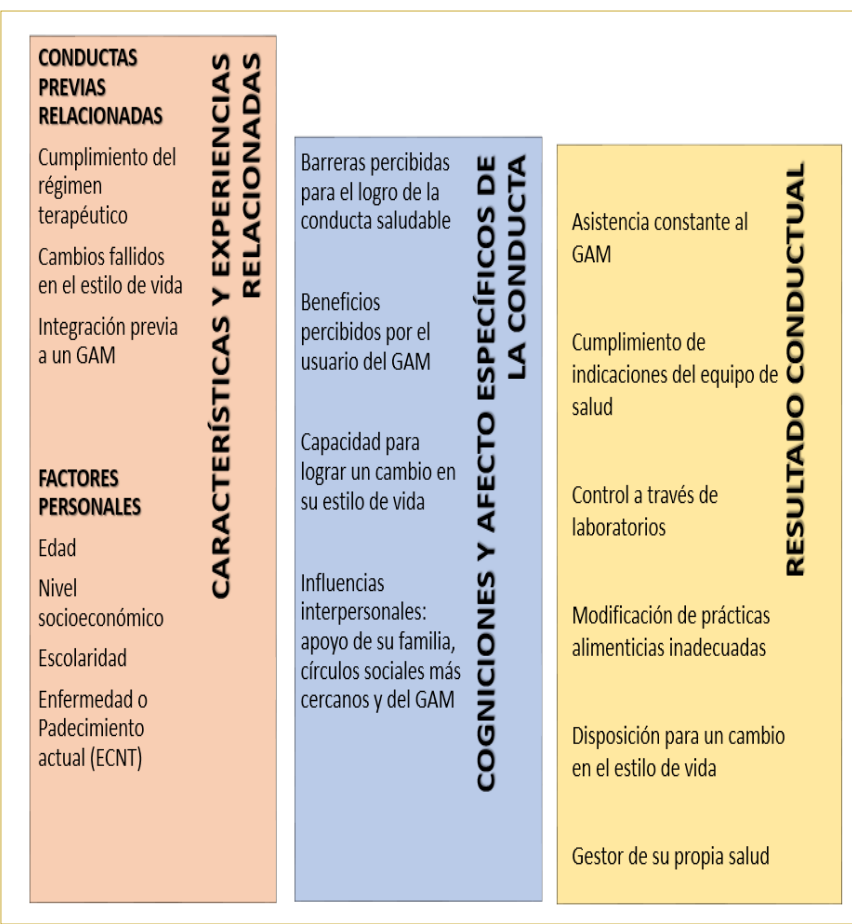

Fuente: Elaboración propia.

\section{Propuesta de planes de cuidados estandarizados para integrantes de grupos de ayuda mutua (PCGAM).}

Para la aplicación del PE, el profesional de enfermería requiere de una base de conocimientos científicos, lo más amplia y variada posible, así como habilidades manuales, intelectuales e interpersonales con la mejor actitud de empatía para con el sujeto de cuidado, así como de mantener el respeto a los valores y creencias del usuario y conservar un estilo de vida saludable.

Para lograr lo anterior, se requiere de un esfuerzo educativo adicional que modifique los hábitos de vida del enfermo, es decir, su alimentación diaria, su actividad física, su cuidado personal, su aprendizaje sobre la enfermedad y su autocontrol, los factores socioculturales que constituyen la base del tratamiento, así como los factores ambientales y personales que inciden en el proceso de cambio que estos enfermos requieren para vivir su vida lo más plenamente posible.

Los planes de cuidados estandarizados para grupos de ayuda mutua son una propuesta que documenta y comunica la situación de salud de las personas que integran los GAM y propone a través de intervenciones generalizadas brindar herramientas de para el logro de conductas promotoras de la salud de forma individualizada, cada caso en particular, con actividades concretas para logar los resultados esperados como puede ser el 
estímulo a la adopción de estilos de vida más saludables y así, prevenir y/o controlar la enfermedad, las complicaciones derivadas de la misma y obtener una mejor calidad de vida.

La propuesta de intervención de enfermería está conformada por 12 diagnósticos de enfermería (Figura 3), 12 resultados esperados y 12 intervenciones de enfermería que conducen al planteamiento de actividades específicas con un enfoque de promoción de la salud dirigidos a los integrantes del GAM a través de Planes de cuidados estandarizados. Para el fin de este artículo sólo se incluyen dos de los planes estandarizados que incluye la propuesta (Figura 4 Y 5).

Figura 3. Etiquetas diagnósticas incluidas en la propuesta de proceso de enfermería para GAM.

\begin{tabular}{|l|}
\hline ETIQUETA DIAGNÓSTICA (NANDA-I) \\
(00162) Disposición para mejorar la gestión de \\
la propia salud \\
\hline (00163) Disposición para mejorar la nutrición \\
\hline $\begin{array}{l}\text { (00068) Disposición para mejorar el bienestar } \\
\text { espiritual }\end{array}$ \\
(00243) Disposición para mejorar la autonomía \\
para la toma de decisiones \\
\hline $\begin{array}{l}\text { (00158) Disposición para mejorar el afronta- } \\
\text { miento }\end{array}$ \\
\hline $\begin{array}{l}\text { (00167) Disposición para mejorar el autocon- } \\
\text { cepto }\end{array}$ \\
\hline (00185) Disposición para mejorar la esperanza \\
\hline (00168) Estilo de vida sedentario \\
\hline (00099) Mantenimiento ineficaz de la salud \\
\hline (00179) Riesgo de nivel de glucemia inestable. \\
\hline (00232) Obesidad y/o (00233) Sobrepeso \\
\hline (00079) Incumplimiento \\
\hline
\end{tabular}

Fuente: Elaboración propia.
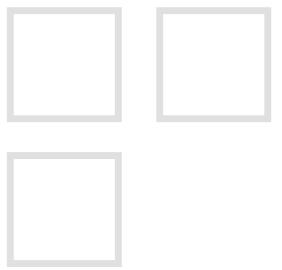


\section{SALUD}

EALUD

CARACTERÍSTICAS DEFINITORIAS: Expresa deseos de mejorar el manejo de los factores de riesgo; expresa deseos de mejorar el manejo de los síntomas, expresa deseos de mejorar el manejo de su enfermedad; expresa deseos de manejar el régimen prescrito; expresa deseos de mejorar las elecciones de la vida diaria para satisfacer los objetivos.

\section{RESULTADOS ESPERADOS (NOC):}

CONOCIMIENTO REGIMEN TERAPEUTICO, CONDUCTA DE CUMPLIMIENTO TERÁPEUTICO, PARTICIPACIÓN EN LAS DECISIONES DE CUIDADOS DE SALUD, CONDUCTA TERÁPEUTICA: ENFERMEDAD O LESIÓN ${ }^{(7)}$.

DEFINICIÓN: ( SE DEFINIRÁ CADA UNO DE LOS RESULTADOS ESPERADOS SELECCIONADOS)

\begin{tabular}{|c|c|c|c|}
\hline INDICADORES: & \multicolumn{2}{|c|}{ PUNTUACIÓN DIANA } & ESCALA DE MEDICIÓN \\
\hline \multirow{7}{*}{$\begin{array}{l}\text { (LISTAR INDICADORES CON LA } \\
\text { ESCALA CORRESPONDIENTE DE } \\
\text { ACUERDO A LOS RESULTADOS } \\
\text { ESPERADOS SELECCIONADOS ) }\end{array}$} & MANTENER A: & AUMENTAR A: & \multirow{2}{*}{1} \\
\hline & \multirow{3}{*}{\multicolumn{2}{|c|}{$\begin{array}{l}\text { Cada indicador tendrá una puntuación } \\
\text { correspondiente a la valoración inicial, } \\
\text { basada en la escala de medición que sir- } \\
\text { ve para evaluar el resultado esperado. }\end{array}$}} & \\
\hline & & & 2 \\
\hline & \multirow{4}{*}{\multicolumn{2}{|c|}{$\begin{array}{l}\text { El objeto de las intervenciones es man- } \\
\text { tener la puntuación e idealmente au- } \\
\text { mentarla. Ambos puntajes sólo pueden } \\
\text { ser determinados en la atención indivi- } \\
\text { dualizada a la persona, familia o comu- } \\
\text { nidad expresada en los registros clínicos } \\
\text { de enfermería. }\end{array}$}} & 3 \\
\hline & & & 4 \\
\hline & & & 5 \\
\hline & & & \\
\hline \multicolumn{4}{|c|}{$\begin{array}{l}\text { INTERVENCIONES (NIC): PROCESO DE ENFERMEDAD, DERIVACIÓN, APOYO FAMILIAR, ORIENTACIÓN AL } \\
\text { SISTEMA DE SALUD }{ }^{(8)} \text {. }\end{array}$} \\
\hline \multicolumn{4}{|c|}{$\begin{array}{l}\text { ACTIVIDADES: (LISTAR ACTIVIDADES CORRESPONDIENTES A LA ETIQUETA DE INTERVENCIÓN SELEC- } \\
\text { CIONADA) }\end{array}$} \\
\hline
\end{tabular}

Fuente: Elaboración propia. 


\section{Figura 5. PLAN DE CUIDADOS ESTANDARIZADO PARA GRUPOS DE AYUDA MUTUA}

ETIQUETA DIAGNOSTICA (NANDA): DISPOSICIÓN PARA MEJORAR LA NUTRICIÓN ${ }^{(6)}$

CARACTERÍSTICAS DEFINITORIAS: Actitud hacia el acto de beber congruente con los objetivos de salud, actitud hacia el acto de comer congruente con los objetivos de salud, consumo de líquidos adecuados, de alimentos adecuados, come regularmente, expresa conocimientos sobre elecciones saludables de líquidos, de alimentos, expresa deseos de mejorar la nutrición, sigue un estándar apropiado para los aportes (Ejemplo: plato del buen comer), seguridad en la preparación de los líquidos, seguridad en la preparación de los alimentos, seguridad en el almacenamiento de los líquidos, de los alimentos.

DOMINIO:

CLASE:

RESULTADOS ESPERADOS (NOC): ESTADO NUTRICIONAL, ENSEÑANZA DE LA NUTRICIÓN (7). DEFINICIÓN: ( SE DEFINIRA CADA UNO DE LOS RESULTADOS ESPERADOS SELECCIONADOS)

\begin{tabular}{|c|c|c|c|}
\hline INDICADORES: & PUNTUAC & ÓN DIANA & ESCALA DE MEDICIÓN \\
\hline \multirow{4}{*}{$\begin{array}{c}\text { (LISTAR INDICADORES CON LA } \\
\text { ESCALA CORRESPONDIENTE DE } \\
\text { ACUERDO A LOS RESULTADOS } \\
\text { ESPERADOS SELECCIONADOS) }\end{array}$} & MANTENER A: & AUMENTAR A: & 1 \\
\hline & \multirow{4}{*}{\multicolumn{2}{|c|}{$\begin{array}{l}\text { Cada indicador tendrá una puntuación co- } \\
\text { rrespondiente a la valoración inicial, basa- } \\
\text { da en la escala de medición que sirve para } \\
\text { evaluar el resultado esperado. } \\
\text { El objeto de las intervenciones es mante- } \\
\text { ner la puntuación e idealmente aumentar- } \\
\text { la. Ambos puntajes sólo pueden ser deter- } \\
\text { minados en la atención individualizada a } \\
\text { la persona, familia o comunidad expresada } \\
\text { en los registros clínicos de enfermería. }\end{array}$}} & 2 \\
\hline & & & 3 \\
\hline & & & 4 \\
\hline (CONSULTAR NOC) & & & 5 \\
\hline \multicolumn{4}{|c|}{ INTERVENCIONES (NIC): GESTIÓN DE LA NUTRICIÓN, CONTROL DE LA NUTRICIÓN ${ }^{(8)}$. } \\
\hline ACTIVIDADES: ( LISTAR ACTIVIDA) & $\begin{array}{r}\text { CORRESPONDIE } \\
\text { (CONSU }\end{array}$ & $\begin{array}{l}\text { A LA ETIQUETA D } \\
\text { NIC) }\end{array}$ & NTERVENCIÓN SELECCIONADA) \\
\hline
\end{tabular}

Fuente: Elaboración propia.

\section{CONCLUSIONES}

La realización de una propuesta de PE para GAM, desde la perspectiva del MPS, ofrece la oportunidad de aportar herramientas teórico- metodológicas para brindar cuidado a usuarios con ECNT como: diabetes mellitus, hipertensión arterial, obesidad y dislipidemias que conforman estos grupos. 
En el área de promoción de la salud, las estrategias educativas tienen como objetivo fomentar la salud en las personas, proporcionar medidas preventivas, elevando el nivel de conocimientos sobre ECNT y sus complicaciones a corto, mediano y largo plazo, así como conseguir que dichos conocimientos sean puestos en práctica y logren el efecto deseado. Sin embargo, las estrategias utilizadas en la mayor parte de los casos no han sido las idóneas, debido posiblemente a que se fomenta la pasividad en el enfermo colocándolo como receptor y consumidor de información sin impactar en su estilo de vida.

Se hace necesaria una educación participativa que considere al enfermo crónico como generador de su propio aprendizaje al establecer un vínculo entre la teoría y la práctica para lograr un efecto en la toma de decisiones sobre hábitos y estilos de vida saludables $y$, como consecuencia, en el control de la enfermedad.

La propuesta de intervención de enfermería para GAM, basada en la perspectiva del MPS no es concluyente, ya que existen otras estructuras o modelos teóricos que pueden ser aplicados y servir de base para la aplicación del PE en el ámbito de primer nivel de atención.

Dado lo anterior y teniendo como meta el bienestar y la calidad de vida de los miembros del GAM, el compromiso del profesional de enfermería es primordial para ejercer la profesión con eficacia, eficiencia y calidez en el cuidado humano brindado.

\section{REFERENCIAS BIBLIOGRÁFICAS}

1. Kuri-Morales P. Transicipon en salud y su impacto en la demanda de los servicios. Gaceta médica de México. 2011; 147: p. 451-4.

2. Cruz M, Pérez M, Jenaro C, Flores N, Segovia M. Necesidad social de formacipon de recursos profesionales para el cuidado: una disyuntiva para la Enfermería profesional. Index de Enfermería. 2010; 19(4): p. 269-73.

3. Franco Z. La promoción de la salud en el marco de la bioética y el desarrollo humano. Hacia la promoción de la salud. 2006; 11: p. 39-49.

4. Raile A, Marriner T. Modelos y teorías en enfermería. 7th ed. Barcelona: Elsevier Mosby; 2011.

5. Aristizábal G, Blanco D, Sánchez A, Ostiguín R. El modelo de promoción de la salud de Nola Pender. Una reflexipon entorno a su comprensión. Enfermería Universitaria. 2011; 8(8): p. 16-23.

6. Herdmann H, Kamitsuru S. Nanda Internacional. Diagnósticos Enfermeros: definiciones y calsificación 2015-2017. Barcelona: Elsevier; 2015.

7. Moorhead S, Johnson M, Maas M, Swanson E. Clasificación de Resultados de Enfermería (NOC): medición de resultados en salud. 5th ed. Barcelona: Elsevier; 2014.

8. Bulechek G, Butcher H, Dochterman J, Wagner C. Clasificación de Intervenciones de Enfermería (NIC). 6th ed. Barcelona: Elsevier; 2014.

9. Álfaro-Lefevre R. Pensamiento crítico y juicio clínico en enfermería. Cuarta ed. Barcelona: Elsevier Masson; 2009.

10. Kozier B, Berman A, Snyder S, Erb G. Fundamentos de enfermería: conceptos, proceso y práctica. 8a Edición. Volumen II. Madrid, España.: PEARSON EDUCACIÓN, S.A.; 2008.

11. Gabaldon B. Los planes de duidados como evaluación escrita de las práctica clínicas. 2008..

12. Beristaín I, Díaz M. Guía de valoración del paciente adulto basado en el modelo de promoción de la salud. Desarrollo Científ Enferm. 2009; 17(6): p. 278-82.

13. Cid P, Merino J, Stiepovich J. Factores biológicos y psicosociales predictores del estilo de vida promotor de salud. Rev Méd Chile. 2006; 134: p. 1491-9.

14. Alligod M. Modelos y teorías en enfermería. 8th ed. Barcelona: Elsevier; 2015. 